$16^{\text {th }}$ International Congress of Metrology, 11012 (2013)

DOI: $10.1051 /$ metrology/201311012

(c) Owned by the authors, published by EDP Sciences, 2013

\title{
Institutional System of Metrology (ISM) of the Federal Electricity Commission (FEC)
}

\author{
Bárbara Montoya-Escobar ${ }^{\text {a1 }}$, María Teresa Santaella-Cópil ${ }^{1}$ \\ ${ }^{1}$ CFE Laboratory of Metrology, GEIC 265 Augusto Rodin, México
}

\begin{abstract}
The activities of metrology in the Federal Electricity Commission are shaped in a system of governance that aims to establish the metrological traceability that will ensure the reliability of the measurements carried out in all substantive processes of construction, generation, transmission and distribution. Benefits: 1. Ensure that commercial transactions of electric power are correct and reliable. 2. Optimize the use of resources with the regulation of the institution metrological infrastructure. 3. Having an institutional metrological system. 4. Having a system metrological of Vanguard that contributes to the positioning of the institution as a world-class company.
\end{abstract}

\section{Introduction}

These policies are established to formalize the structure, roles and responsibilities of the institutional system of metrology of the FEC (ISMFEC) and is created to assist functions in the field of metrology in the Organization and orients its interrelationship with metrology at national and international level, pursuant to the Federal law on metrology and standardization and its regulation as well as provisions of the institutional regulations.

The activities of metrology in the FEC, is shaped into a system of governance which aims to establish the metrological traceability that will ensure the reliability of the measurements carried out in all the substantive processes of construction, generation, transmission and distribution.

The ISMFEC has as main purpose to contribute to the Mission of the Federal Electricity Commission:

Ensure, within a framework of competition and technologically, updated the service of electricity, in terms of quantity, quality and price, with the adequate diversification of energy sources.

- Optimize the use of your physical, commercial infrastructure and human resources.

- Provide an excellent service to our customers.

- Protect the environment, promote social development and respect the values of populations where the electrification works are located.

The functional areas of FEC involved and their relationship with the measurements are:

1. Projects and construction:
- Determination of the design parameters of the generation, transformation, transmission and distribution of electric power facilities.

- Determination of parameters for the assurance of the quality applied to construction.

- Measurements for monitoring the safety of the civilian infrastructure of the institution.

2. Generation:

- Measurement in the points of reception and consumption of energy input in generation plants.

- Use of instruments and measuring systems to maintain optimum and safe operation the generation processes.

- Measurement of electric power delivery points. 3. Transmission:

- Measurement at the points of reception, delivery and consumption of electrical energy and power quality.

- Utilization of monitoring and test equipment to maintain optimum and safe operation transmission processes.

4. Distribution:

- Flow measurement of electrical energy in the distribution network, exchanges of energy with other areas of the entity, transactions of electricity with generators individuals and customers, as well as power quality.

5. LATEM (Laboratory testing of equipment and materials):

- Maintenance of the metrological traceability for the ISMFEC.

- Maintenance of the metrological traceability of the test laboratories of the LATEM.

This document shows the vision, mission, objectives, benefits and structure of the ISMFEC, as well as generals of the system policies and the functions and

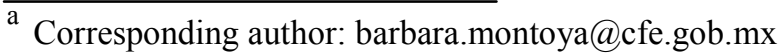


responsibilities of those involved in it, required for the proper operation of the ISMFEC.

\section{Benefits of the ISMFEC for the FEC}

- Ensure that commercial electricity transactions are correct and reliable, in accordance with the laws and regulations.

- Achieving the satisfaction of customers through recognition of the technical competence of the institution to carry out calibrations, measurements and tests.

- Technically regular purchases of equipment for inspection, measurement and testing, making the institution.

- Optimize the use of resources with the regulation of the institution of metrological infrastructure.

- Having an institutional metrological system allowing to ensure the reliability of the measurements in the substantive processes of the FEC.

- A system of metrology of the art that contributes to the positioning of the institution as a world-class company.

\section{Application}

This document is enforceable in all areas involved in the structure of the ISMFEC, including the representatives of all areas designated in the Coordinating Committee and the personnel that work in the laboratories of the ISMFEC of FEC.

\section{Mission}

The Mission of the ISMFEC is as follows: the institutional system of metrology of FEC (ISMFEC), ensures the reliability of the measurements carried out in the different processes, through a network of formally recognized laboratories, which are equipped with advanced technology infrastructure and technically competent personnel, adapting continuous and timely manner to emerging measurement needs, contributing to the positioning of the institution as a world-class company.

\section{Vision}

The vision of the ISMFEC is as follows: to be recognized in the FEC and the national and international level as a metrological management system which ensures the reliability of the measurements, in accordance with the regulations of the specialty, exceeding the expectations of our customers.

\section{Goals}

- Establish policies to operate the network of metrology laboratories according to the needs of FEC, maintaining the chain of traceability allowing to ensure the reliability of the measurements, operating under the existing standard NMX-EC-17025-IMNC [1].
- Technologically updated the laboratory network.

- Continuously improve the skills of the staff that operates the network of laboratories.

- To promote in the FEC metrological culture.

\section{General policies}

- All work in the field of metrology at the institutional level should be made in accordance with the established policies of operation here.

- It is responsible for the operating directions, investment funded and modernization projects provide the human and financial, required resources for the operation of the ISMFEC.

- It is responsible for establishing and setting the policies and guidelines of the ISMFEC operation, is the Coordinating Committee of the ISMFEC.

- The members of the Committee Coordinator of the system, are officially designated by the owner of the area as representatives of each of the branches I managers in accordance with the organizational structure.

- The Coordinating Committee has the authority to establish technical sub-committees for solving metrological issues I drafting documents related to the system.

- The Coordinating Committee approval sends policies to the Board of Directors, for its subsequent implementation by members of the ISMFEC areas.

- The Coordinating Committee should develop and establish the rules of operation of the Coordinating Committee.

- The current versions of these policies, guidelines and operating procedures will be available for consultation on the website of the institutional system of metrology.

- The magnitudes that metrologically are met, in accordance with the requirements of the process, should have the formal approval of the Deputy Director of the corresponding area or in your case of the Manager of the nuclear power plant.

\section{Organization of the ISMFEC}

Organizational structure of the ISMFEC laboratories. Addresses that make up the ISMFEC should formally include within its structure organic and administrative, to each of the laboratories of secondary and tertiary, to assign the financial, human and material resources adequate and sufficient for its operation, through a cost for laboratory Center.

Financial, human and material resources.

The laboratory must have managerial staff and technician who has the authority and adequate financial, material resources and sufficient for its operation. The minimum requirements for the operation of the laboratory, are defined in the procedure of financial resources, human and material.

Metrological traceability. 
The metrological traceability through a chain unbroken and documented calibration, be sure to know the uncertainty of measurements, in accordance with the procedure of traceability of measurements, current.

The reference laboratory should implement and maintain calibrated reference standards of the FEC, with traceability of national employers maintained by the National Center of Metrology (NACEM) to international standards.

In order to maintain the necessary traceability any acquisition patterns and equipment, in different workplaces, they must take into account uncertainties required by the process and submit your proposal to the laboratory from the upper level of the chain of traceability, for his technical support.

All laboratories should calibrate your patterns with the laboratory's top level in accordance with the Charter of traceability and in case of not having the required pattern or for cause of the higher level laboratory, reference laboratory or another accredited external laboratory may be used.

The calibration of instrumentation in the FEC process, must be carried out with the team pattern, personnel and procedure that corresponds. In case of not having available the pattern or for cause of the top-level lab, you can use another accredited external laboratory.

Management system.

The ISMFEC laboratories should establish, implement and maintain a management system appropriate to the scope of its activities, which complies with the requirements of the norm NMX-EC-17025-IMNC [1], existing.

Laboratory accreditation to the MAE (Mexican Accreditation Entity).

Accordance with the provisions of the Federal law of metrology and standardization, second title, chapter III, of the compulsory measurement of transactions, articles 15 and 17 and in its rules of procedure, in its second title, chapter III, articles 8 and 14; all laboratories that have quantities or variables involved in commercial transactions, are required to have the appropriate accreditation in quantities or variables related to these provisions.

\section{By comparisons of measurements interlaboratory} proficiency testing.

In the ISMFEC testing proficiency established by measurements, interlaboratory comparisons to ensure the competence of laboratories to produce technically valid results, according to the Mexican norm NMX-EC-043-1 [1], force, according to proficiency among laboratories, existing testing procedure.

\section{Uncertainty.}

The determination of the uncertainty of measurement for each step of the chain of traceability of the ISMFEC, must be estimated by each laboratory applying the methods defined in the Mexican standard NMX-CH-140-IMNC [3], force, according to the procedure for the determination of the uncertainty, existing.

\section{Competencies of staff.}

The head of the laboratory must ensure the competence of anyone operating laboratory instruments and equipment, perform calibrations, evaluate results and sign reports of calibration, according to the procedures laid down in the corresponding Integral management system. Procedure to ensure the competence of staff, recent.

\section{Conformity assessment of laboratories of the ISMFEC.}

In order to ensure the technical validity, the quality of the offered services and verify that the operations of laboratories continue complying with the requirements of the management system and of the NMX-EC-17025IMNC [1], force, should be periodically visits of conformity assessment, according to the procedure of the conformity assessment on the existing standard NMX-EC17025-IMNC [1].

Coordinates the program of secondary laboratories assessment visits the reference laboratory, which in conjunction with the trained and qualified personnel from all areas carry out such assessments, according to the evaluators qualification procedure, force.

Relationship with the Integral system of management of the addresses of the ISMFEC.

The representatives of the Coordinating Committee should establish and maintain the metrological management system with Integral management system of each of the directions of the ISMFEC, linked to ensure the operation of both systems in a coordinated and efficient manner.

\section{Roles and responsibilities}

\section{Of the Coordinating Committee of the ISMFEC.}

The roles and responsibilities of the Coordinating Committee are established in the rules of the operation of the Coordinating Committee of the ISMFEC (CCISMFEC).

In the areas of the FEC.

The roles and responsibilities of the areas of the FEC, are established in the rules of operation of the Coordinating Committee of the ISMFEC.

Designate officially members of the Coordinating Committee (members and alternates) who will be as representatives of the branches I managements, for the development of the activities of metrology in the FEC.

Facilitate the participation of the staff of the laboratories in the technical sub-committees for the preparation, review and approval of the operating procedures of the ISMFEC, making sure that involved staff, trained and experienced in the subject matter, designated official for each of the topics.

Implement and disseminate the documents of the ISMFEC in their areas for its implementation. 
Promote the culture of metrology in the field of your area.

Of the technical subcommittees.

The roles and responsibilities of the technical subcommittees are established in the rules of the operation of the Coordinating Committee of the ISMFEC (CCISMFEC).

\section{Reference regulations}

[1] NMX-EC-17025-IMNC. General requirements for the competence of testing and calibration, current laboratories. [2] NMX-EC-043-1. Testing proficiency by interlaboratory comparisons - part 1. Development and operation of proficiency, existing testing programs.

[3] NMX-CH-140-IMNC. Guide to the expression of uncertainty in measurements, current.

- Federal law on metrology and standardization and its existing regulations (LFMN)

- Service law public power and its current Regulation (LSPEE).

- NOM-008-SCFI. General unit of measure, existing system

- NMX-Z-055-IMNC. Vocabulary international of metrology - basic and general concepts associated terms (VIM), current. 